\title{
Vibrações, Ruído e Hipoacusia: associação fisiopatológica ou não?
}

rpso.pt/vibracoes-e-hipoacusia-associacao-fisiopatologica-ou-nao/

Santos M, Almeida A. Lopes C. Oliveira T. Vibrações e Hipoacusia: associação fisiopatológica ou não? Revista Portuguesa de Saúde Ocupacional on line. 2020, volume 9, 1-11. DOI: 10.31252/RPSO.25.04.2020

VIBRATIONS, NOISE AND HYPOACUSIA: PHYSIOPATHOLOGICAL ASSOCIATION OR NOT?

TIPO DE ARTIGO: Scoping Review

Autores: Santos M(1), Almeida A(2), Lopes C(3), Oliveira T(4).

\section{RESUMO}

\section{Introdução/ enquadramento/ objetivos}

As vibrações e o ruido são dois fatores de risco laborais razoavelmente prevalentes e, em muitas situações, presente em conjunto. Caso seja real uma associação fisiopatológica entre ambos, será bastante relevante comprovar tal, de forma a reajustar, se necessário, os limites das vibrações e ruido considerados seguros.

\section{Metodologia}

Trata-se de uma Scoping Review, iniciada através de uma pesquisa realizada em setembro de 2019, nas bases de dados "CINALH plus with full text, Medline with full text, Database of Abstracts of Reviews of Effects, Cochrane Central Register of Controlled Trials, Cochrane Database of Systematic Reviews, Cochrane Methodology Register, Nursing and Allied Health Collection: comprehensive, MedicLatina, Academic Search Ultimate, Science Direct, SCOPUS e RCAAP".

\section{Conteúdo}

Alguns investigadores quantificaram maiores perdas de audição em trabalhadores simultaneamente expostos a ruido e vibrações; contudo, trabalhadores expostos a vibrações provenientes de instrumentos de trabalho, geralmente estão expostos também a níveis mais elevados de ruido.

Caso exista uma ligação fisiopatológica entre estes dois fatores de risco laborais, acredita-se que esta possa incidir na teoria da vasoconstrição simpática (observada no síndroma dos dedos brancos) e que também poderá danificar a cóclea, produzindo danos isquémicos a nível das células ciliadas.

Outra hipótese destaca que algumas ferramentas de trabalho poderão originar vibrações capazes de atingir diretamente o ouvido interno, por condução óssea (sobretudo a nível temporal).

\section{Conclusões}

A generalidade dos estudos consultados não apresentava metodologia muito robusta, que permita avaliar com rigor se a hipoacusia mais intensa em indivíduos simultaneamente expostos a vibrações resulta de uma verdadeira associação fisiopatológica, ou se se trata apenas de um enviesamento estatístico.

Seria interessante que equipas de saúde ocupacional a exercer em clientes com trabalhadores simultaneamente expostos a estes dois fatores de risco (e, se possível, com combinações de intensidades diferentes) investigassem o tema, de forma a se produzir conhecimento capaz de potenciar a saúde e segurança ocupacionais.

PALAVRAS/ EXPRESSÕES- CHAVE: hipoacusia, vibrações, saúde ocupacional e medicina do trabalho.

\section{ABSTRACT}




\section{Introduction / framework / objectives}

Vibrations and noise are two reasonably prevalent occupational risk factors and, in many situations, present together. If a pathophysiological association between them is real, it will be very relevant to verify this, in order to readjust, if necessary, the vibration and noise limits considered safe.

\section{Methodology.}

This is an Scoping Review, initiated by a September 2019 survey of the "Cochrane Central Register of Controlled Trials databases, CINALH plus with full text, Database of Abstracts of Reviews of Effects, Cochrane Database of Systematic Reviews, Cochrane Methodology Register, Nursing and Allied Health Collection: Comprehensive, MedicLatina, Academic Search Ultimate, Science Direct, SCOPUS and RCAAP."

\section{Content}

Some researchers have quantified higher hearing losses in workers simultaneously exposed to noise and vibrations; however, workers exposed to vibration from work instruments are generally also exposed to higher levels of noise.

If there is a pathophysiological link between these two occupational risk factors, it is believed that this may be related to the theory of sympathetic vasoconstriction (observed in white-finger syndrome) and may also damage the cochlea, producing ischemic damage to the hair cells.

Another hypothesis points out that some work tools may give rise to vibrations that can reach the inner ear directly, through bone conduction (especially at the temporal level).

\section{Conclusions}

Most of the studies consulted did not have a very robust methodology that allows a rigorous evaluation of whether the most intense hypoacusis in individuals simultaneously exposed to vibration results from a true pathophysiological association, or if it is only a statistical bias.

It would be interesting for occupational health teams to investigate on clients with workers simultaneously exposed to these two risk factors (and, if possible, with combinations of different intensities), in order to produce knowledge capable of enhancing occupational health and safety.

KEY WORDS / EXPRESSIONS: hypoacusis, vibrations, occupational health and occupational medicine.

\section{INTRODUÇÃO}

As vibrações e o ruido são dois fatores de risco laborais razoavelmente prevalentes e, em muitas situações, presentes em conjunto para alguns trabalhadores. Caso seja real uma associação fisiopatológica entre ambos, será bastante relevante comprovar tal, de forma a reajustar, se necessário, os limites de vibrações e ruido considerados seguros.

\section{METODOLOGIA}

Em função da metodologia PICo, foram considerados:

-P (population): trabalhadores expostos simultaneamente a vibrações e ruido.

-I (interest): eventual associação fisiopatológica entre as vibrações e o ruído

-C (context): saúde ocupacional nas empresas com postos de trabalho com vibrações e ruido.

Pergunta protocolar: A eventual associação entre hipoacusia e vibrações assenta em bases fisiopatológicas reais ou resulta apenas do enviesamento secundário ao facto de muitas máquinas com vibrações apresentarem também ruido considerável?

Foi realizada uma pesquisa em setembro de 2019 nas bases de dados "CINALH plus with full text, Medline with full text, Database of Abstracts of Reviews of Effects, Cochrane Central Register of Controlled Trials, Cochrane Database of Systematic Reviews, Cochrane Methodology Register, Nursing and Allied Health Collection: comprehensive, MedicLatina, Academic Search Ultimate, Science Direct, SCOPUS e RCAAP".

No quadro 1 podem ser consultadas as palavras/ expressões- chave utilizadas nas bases de dados. No quadro 2 estão resumidas as caraterísticas metodológicas dos artigos selecionados. 


\section{CONTEÚDO}

Alguns investigadores quantificaram maiores perdas de audição em trabalhadores simultaneamente expostos a ruido e vibrações [1], nomeadamente entre os que apresentavam síndroma de dedos brancos [2] [3] [4] [5], sobretudo (mas não só) para frequências sonoras elevadas, nomeadamente na ordem de duas vezes mais [2]. $O$ fenómeno de Raynaud consiste na vasoconstrição sanguínea digital, que justifica a alteração na coloração tipicamente associada [4] [5]; a vasoconstrição pode ser secundária ao stress, frio e/ ou vibrações ou então primária [4]. A exposição a vibrações pode originar a síndroma de vibração mão-braço, que inclui (além das questões vasculares já mencionadas) eventuais alterações neurológicas e músculo-esqueléticas. Indivíduos com síndroma de dedos brancos associados a vibrações, apresentam hipoacusia mais intensa; ou seja, alguns acreditam que a síndroma de dedos brancos pode servir como um "marcador de suscetibilidade" para a hipoacusia [5].

Alguns investigadores salientam que trabalhadores mais expostos a vibrações mão-braço geralmente utilizam máquinas mais ruidosas com maior proximidade, daí que apresentem usualmente maiores perdas auditivas [2]. Ou seja, trabalhadores expostos a vibrações provenientes de instrumentos de trabalho, geralmente estão expostos também a níveis mais elevados de ruido [1] [4] [5].

Caso exista uma ligação fisiopatológica entre estes dois fatores de risco laborais, acredita-se que esta possa incidir na teoria da vasoconstrição simpática (observada no síndroma dos dedos brancos) e que também poderá danificar a cóclea [1] [2] [4], produzindo danos isquémicos a nível das células ciliadas [2] [4]. Os efeitos das vibrações na cóclea poderão estar dependentes da frequência vibratória, intensidade, tempo de exposição, postura corporal e variabilidade individual [6].

Outra hipótese destaca que algumas ferramentas de trabalho poderão originar vibrações capazes de atingir o ouvido interno, por condução óssea (sobretudo a nível temporal), segundo investigações efetuadas em animais [2].

Estudos em animais também sugerem uma maior perda de audição com a exposição simultânea a vibrações, ainda que tal não seja consensual [1]. Na realidade, na bibliografia consultada (para humanos) os investigadores não são consensuais relativamente às vibrações potenciarem ou não a hipoacusia [5] [6].

\section{DISCUSSÃO/ CONCLUSÃO}

A generalidade dos estudos consultados não apresentou consistência metodológica, que permita avaliar com rigor se a hipoacusia mais intensa em indivíduos simultaneamente expostos a vibrações e ruido resulta de uma verdadeira associação fisiopatológica (baseada na vasoconstrição), ou se se trata apenas de um enviesamento estatístico, secundário ao facto de máquinas de trabalho com vibrações geralmente também implicarem exposição a mais decibéis.

Seria interessante que equipas de saúde ocupacional com amostras de trabalhadores simultaneamente expostos a estes dois fatores de risco (e, se possível, com combinações de intensidades diferentes) investigassem o tema, de forma a se produzir conhecimento capaz de potenciar a saúde e segurança ocupacionais.

\section{CONFLITOS DE INTERESSE, QUESTÕES ÉTICAS E/OU LEGAIS}

Nada a declarar.

\section{AGRADECIMENTOS}

Nada a declarar 
[1] Pettersson H, Burstrom L, Hagberg M, Lundstrom R, Nilsson T. Noise and hand-arm vibration exposure in relation to the risk of hearing loss. Noise \& Health. 2012, 14(59), 159- 165. DOI: 10.4103/1463-1741.9988

[2] Turcot A, Girard S, Courteau M, Baril J, Larocque R. Noise- induced hearing loss and combined noise and vibration exposure. Occupational Medicine. 2015, 65, 238- 244. DOI: 10. 1093/occmed/Kqu214

[3] Petterson H, Burstrom L, Hagbert M, Lundstrom R, Nilsson T. Risk of hearing loss among workers with vibrationinduced white fingers. American Journal of Industrial Medicine. 2014, 57(12), 1311-1318. DOI: 10.1001/ajim.22368

[4] Petterson H, Burstrom L, Nilsson T. Raynaud's phenomena among men and women with noise. Induced hearing loss in relation to vibration exposure. Noise \& Health. 2014, 16(69), 89- 94. DOI: 10.4103/1463-1741.132087

[5] House R, Sauvé J, Jiang D. Noise-induced hearing loss in construction workers being assessed for hand-arm vibration syndrome. Canadian Journal of Public Health. 2010, 101(3), 226- 229.

[6] Najarkola S, Khavanin A, Mirzaei R, Salehnia M, Muhammadnejad A. Cochlear damage caused by vibration exposure. Iranian Red Crescent Medical Journal. 2013, 15(9), 771- 774. DOI: 10. 5812/ircmj.5369

Quadro 1: Pesquisa efetuada

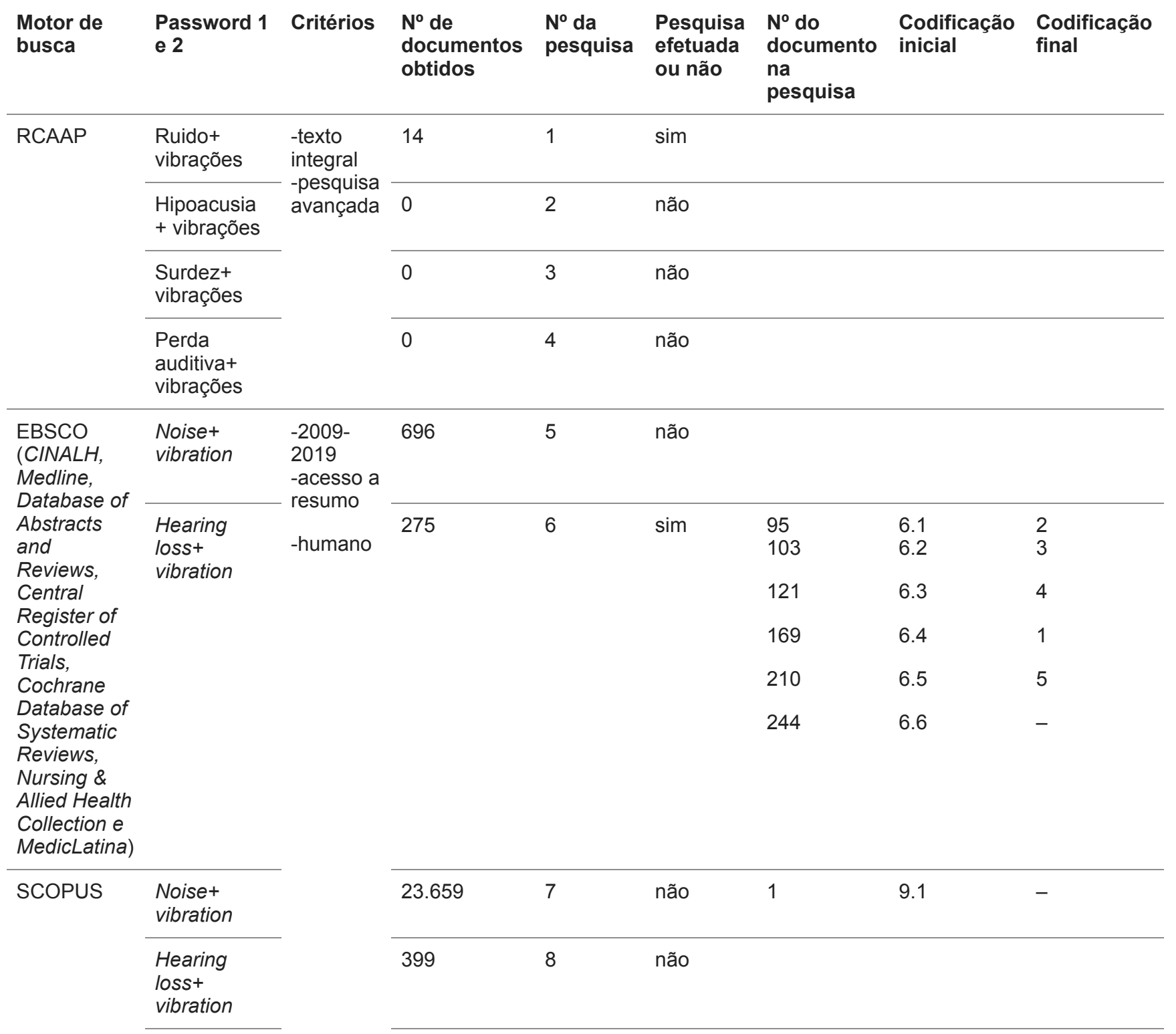




$$
\begin{aligned}
& \text { and } \\
& \text { occupational }
\end{aligned}
$$

\begin{tabular}{llll}
\hline $60 \quad \operatorname{sim}$ & 1 & 9.1 & - \\
11 & Alemão & - \\
25 & $=6.1$ & - \\
26 & $=6.1$ & - \\
30 & $=6.2$ & - \\
31 & $=6.3$ & - \\
35 & 9.2 & - \\
42 & 9.3 & 6 \\
44 & $=6.4$ & -
\end{tabular}

\begin{tabular}{|c|c|c|c|c|c|}
\hline 11.385 & 10 & não & & & \\
\hline 301 & 11 & não & & & \\
\hline \multirow[t]{5}{*}{49} & 12 & $\operatorname{sim}$ & $\begin{array}{l}1 \\
2\end{array}$ & $\begin{array}{l}=9.1 \\
=6.1\end{array}$ & - \\
\hline & & & 6 & $=6.3$ & - \\
\hline & & & 9 & $=6.4$ & - \\
\hline & & & 39 & 12.1 & - \\
\hline & & & 45 & $=6.1$ & - \\
\hline
\end{tabular}

\begin{tabular}{ll}
\hline $\begin{array}{l}\text { Academic } \\
\text { Search } \\
\text { Ultimate }\end{array}$ & $\begin{array}{l}\text { Noise+ } \\
\text { vibration }\end{array}$ \\
\cline { 2 - 2 } & $\begin{array}{l}\text { Hearing } \\
\text { loss+ } \\
\text { vibration }\end{array}$ \\
& $\begin{array}{l}\text { and } \\
\text { occupational }\end{array}$
\end{tabular}

\begin{tabular}{|c|c|c|}
\hline Artigo & $\begin{array}{l}\text { Tipo de } \\
\text { estudo }\end{array}$ & Resumo \\
\hline 1 & Coorte & $\begin{array}{l}\text { Este documento sueco teve como objetivo investigar a eventual associação entre a hipoacusia } \\
\text { e as vibrações mão-braço, numa amostra de } 189 \text { indivíduos identificados como expostos a esta } \\
\text { última questão. Concluiu-se que a associação foi positiva. }\end{array}$ \\
\hline 2 & Coorte & $\begin{array}{l}\text { Este trabalho canadiano analisou a suposta associação entre a perda de audição e a } \\
\text { exposição a vibrações, através da análise de quase } 60.000 \text { trabalhadores. Os autores } \\
\text { verificaram que aqueles com síndroma de dedos brancos apresentavam pior audição. }\end{array}$ \\
\hline 3 & Coorte & $\begin{array}{l}\text { Os autores deste artigo sueco avaliaram o risco de hipoacusia em trabalhadores que também } \\
\text { apresentavam a síndroma de dedos brancos induzida por vibrações, numa amostra de } 184 \\
\text { indivíduos e ao longo de } 21 \text { anos de seguimento. Verificaram que estes apresentavam um risco } \\
\text { de perda de audição } 2,3 \text { vezes superior. }\end{array}$ \\
\hline 4 & $\begin{array}{l}\text { Coorte } \\
\text { retrospetivo }\end{array}$ & $\begin{array}{l}\text { Esta investigação sueca pretendeu avaliar a relação entre a síndroma de dedos brancos/ } \\
\text { Raynaud e a perda de audição, entre } 342 \text { trabalhadores com hipoacusia laboral. Os autores } \\
\text { consideraram provável a associação. }\end{array}$ \\
\hline 5 & $\begin{array}{l}\text { Observacional } \\
\text { analítico }\end{array}$ & $\begin{array}{l}\text { Neste trabalho canadiano usaram-se trabalhadores do setor da construção com exposição a } \\
\text { vibrações mão- braço, seguidos em determinada instituição de saúde. Dos } 191 \text { elementos, } 169 \\
\text { aceitou fazer a audiometria. A associação pareceu positiva, mesmo controlando o eventual } \\
\text { enviesamento do número de anos de trabalho. }\end{array}$ \\
\hline 6 & Experimental & $\begin{array}{l}\text { Este documento iraniano pretendeu avaliar as eventuais alterações cocleares secundárias às } \\
\text { vibrações numa amostra de doze coelhos albinos. Os autores concluíram que as vibrações } \\
\text { estavam associadas aos danos atrás mencionados. }\end{array}$ \\
\hline
\end{tabular}

Quadro 2: Caraterização metodológica dos artigos selecionados 


\section{(1)Mónica Santos}

Licenciada em Medicina; Especialista em Medicina Geral e Familiar; Mestre em Ciências do Desporto; Especialista em Medicina do Trabalho e Doutoranda em Segurança e Saúde Ocupacionais, na Faculdade de Engenharia da Universidade do Porto. Presentemente a exercer nas empresas Medicisforma, Servinecra, Securilabor e Medimarco; Diretora Clínica da empresa Quercia; Diretora da Revista Portuguesa de Saúde Ocupacional on line. Endereços para correspondência: Rua Agostinho Fernando Oliveira Guedes, 42, 4420-009 Gondomar. E-mail: s_monica_santos@hotmail.com

\section{(2)Armando Almeida}

Enfermeiro Especialista em Enfermagem Comunitária, com Competência Acrescida em Enfermagem do Trabalho.

Doutorado em Enfermagem; Mestre em Enfermagem Avançada; Pós-graduado em Supervisão Clínica e em Sistemas de Informação em Enfermagem; Professor Auxiliar Convidado na Universidade Católica Portuguesa, Instituto da Ciências da Saúde - Escola de Enfermagem (Porto) onde Coordena a Pós-Graduação em Enfermagem do Trabalho; Diretor Adjunto da Revista Portuguesa de Saúde Ocupacional on line. 4420-009 Gondomar. E-mail: aalmeida@porto.ucp.pt

\section{()ㅡ)Catarina Lopes}

Licenciada em Enfermagem, desde 2010, pela Escola Superior de Saúde Vale do Ave. A exercer funções na área da Saúde Ocupacional desde 2011 como Enfermeira do trabalho autorizada pela Direção Geral de Saúde, tendo sido a responsável pela gestão do departamento de Saúde Ocupacional de uma empresa prestadora de serviços externos durante 7 anos. Atualmente acumula funções como Enfermeira de Saúde Ocupacional e exerce como Enfermeira Generalista na SNS24. Encontra-se a frequentar o curso Técnico Superior de Segurança do Trabalho. 4715-028. Braga. Email: catarinafflopes@gmail.com

\section{(4)Tiago Oliveira}

Licenciado em Enfermagem pela Universidade Católica Portuguesa. Frequenta o curso de Técnico Superior de Segurança no Trabalho. Atualmente exerce a tempo inteiro como Enfermeiro do Trabalho. No âmbito desportivo desenvolveu competências no exercício de funções de Coordenador Comercial na empresa Academia Fitness Center, assim como de Enfermeiro pelo clube de futebol União Desportiva Valonguense. 4435-718 Baguim do Monte. E-mail:

tiago_sc16@hotmail.com 